\title{
Epidermal Neural Crest Stem Cell (EPI-NCSC)—Mediated Recovery of Sensory Function in a Mouse Model of Spinal Cord Injury
}

\author{
Yao Fei Hu • Krishnaj Gourab • Clive Wells • \\ Oliver Clewes • Brian D. Schmit • Maya Sieber-Blum
}

Published online: 23 April 2010

(C) The Author(s) 2010. This article is published with open access at Springerlink.com

\begin{abstract}
Here we show that epidermal neural crest stem cell (EPI-NCSC) transplants in the contused spinal cord caused a $24 \%$ improvement in sensory connectivity and a substantial recovery of touch perception. Furthermore we present a novel method for the ex vivo expansion of EPINCSC into millions of stem cells that takes advantage of the migratory ability of neural crest stem cells and is based on a new culture medium and the use of microcarriers. Functional improvement was shown by two independent methods, spinal somatosensory evoked potentials (SpSEP) and the Semmes-Weinstein touch test. Subsets of transplanted cells differentiated into myelinating oligodendrocytes. Unilateral injections of EPI-NCSC into the lesion of midline contused mouse spinal cords elicited bilateral improvements. Intraspinal EPI-NCSC did not migrate laterally in the spinal cord or invade the spinal roots and dorsal root ganglia, thus implicating diffusible factors. EPINCSC expressed neurotrophic factors, angiogenic factors, and metalloproteases. The strength of EPI-NCSC thus is that they can exert a combination of pertinent functions in the contused spinal cord, including cell replacement,
\end{abstract}

\footnotetext{
Y. F. Hu • C. Wells

Department of Cell Biology, Neurobiology and Anatomy,

Medical College of Wisconsin,

Milwaukee, WI 53226, USA

K. Gourab • B. D. Schmit

Department of Biomedical Engineering, Marquette University, Milwaukee, WI 53233, USA

O. Clewes $\cdot$ M. Sieber-Blum $(\square)$

Institute of Human Genetics and Northeast England Stem Cell Institute, Centre for Life, Central Parkway, Newcastle University, Newcastle upon Tyne NE1 3BZ, UK

e-mail: maya.sieber-blum@ncl.ac.uk
}

neuroprotection, angiogenesis and modulation of scar formation. EPI-NCSC are uniquely qualified for cellbased therapy in spinal cord injury, as neural crest cells and neural tube stem cells share a higher order stem cell and are thus ontologically closely related.

Keywords Stem cell $\cdot$ Neural crest . Epidermal neural crest stem cell · EPI-NCSC - Spinal cord - Spinal cord injury . Somatosensory evoked potential $\cdot$ Semmes-Weinstein touch test

\section{Introduction}

The aim of this study was to determine whether EPI-NCSC grafts elicit improvement in the connectivity of sensory pathways in the contused spinal cord. EPI-NCSC are multipotent remnants of embryonic neural crest stem cells in a postnatal location, the bulge of hair follicles $[1,2]$. Ontologically, neural crest cells are closely related to neural tube cells, as both are descendents of the same higher order stem cell [3]. Previously we showed that intraspinal EPINCSC survive in large numbers, integrate into the spinal tissue, and that different subsets of cells express markers for neurons, including GABAergic neurons, and for oligodendrocytes but not astrocytes or Schwann cells, and that the scar is vascularized [4]. Importantly, as adult stem cells intraspinal EPI-NCSC did not form tumors or teratomas in the spinal cord [4]. The current study was aimed at determining whether these advantageous characteristics of intraspinal EPI-NCSC lead to functional improvement and at elucidating some of the underlying mechanisms.

In treating spinal cord injury, four issues need to be addressed in concert, that is (1) cell replacement, (2) neurotrophic support, (3) neutralizing inhibitory factors 
that block neuronal regeneration and (4) attenuating the inflammatory response [5]. Thus, combination therapies are likely to constitute future treatments (see e.g., [6]). Olfactory ensheathing cells (e.g., [7, 8]) umbilical blood stem cells (e.g. [9]) and mesenchymal stem cells (e.g., [10]) are adult stem cells that have shown promise in treating spinal cord injury. Progress in functional improvement by modulating the glial scar has been made by systemic application of anti-Nogo-A antibodies (see e.g. [6]) or with chondroitinase treatment [11], while intraspinal delivery of neurotrophic factors have been found to lead to neuronal sparing and targeting (e.g., [12, 13]). The strength of EPINCSC is that they fulfill at least three of the above requirements simultaneously, that is cell replacement, neurotrophic support and the ability to degrade extracellular matrix molecules, which form a barrier for the regeneration of projection neurons.

\section{Material and Methods}

\section{Animals}

Wild type C57BL6/J and C57BL/6-TgN(ACTbEGFP)1Osb [14] mice, which express enhanced green fluorescent protein (EGFP) ubiquitously, were purchased from The Jackson Laboratory (Bar Harbor, ME). Mice were maintained, bred, euthanized and surgery was performed at the Transgenic Mouse Facility of the Medical College of Wisconsin according to the guidelines of the US Public Health Service's Policy of Humane Care and Use of Animals, the Guide for the Care and Use of Laboratory Animals and with the approval of the Institutional Animal Care and Use Committee of the Medical College of Wisconsin.

\section{Bulge Explants}

Bulge explants from whisker follicles of 10-12 week-old C57BL/6-TgN(ACTbEGFP)1Osb mice were prepared exactly as described previously $[1,2,15,16]$. Briefly, whisker follicles were dissected, cleaned, cut first longitudinally and subsequently transversely below and above the bulge region. The bulges were then rolled out of the capsule and placed into collagen-coated culture plates where they adhered to the substratum within one hour. Three to 4 days post-explantation, cells started to emigrate from the bulge explants onto the culture substratum. The culture medium consisted of $85 \%$ Alpha-modified MEM, $10 \%$ fetal calf serum and 5\% day-11 chick embryo extract. Three to four days post-onset of emigration, bulges were removed and the adhering cells detached by trypsin treatment and subsequently subcultured.
Spinal Cord Injury and Cell Grafts

Mice were anesthetized by intraperitoneal injection of $1 \mathrm{mg} / \mathrm{kg}$ Domitor (Pfizer Animal Health, New York, NY 10017) and $75 \mathrm{mg} / \mathrm{kg}$ Ketamine (BEDFORD Laboratories, Bedford, OH 44146). Surgery and spinal cord injury were performed under sterile conditions as described previously [4] with the modification that spinal cord injury was performed on the midline, rather than on the left side of the spinal cord, in order to injure both sides equally. Briefly, a laminectomy was performed at thoracic level 8 (T8) and moderate spinal cord injury was performed at the midline with a modified Newton meter [4] at 12 centi-Newtons $(\mathrm{cN})$ for $3 \mathrm{~s}$. Subsequently, in vitro expanded EPI-NCSC were aspirated into a drawn capillary tube, the tip placed onto the dura mater with a micromanipulator and injected $1 \mathrm{~mm}$ deep into the left side of the spinal cord over a 4-5 $\mathrm{min}$ time span each. Two injections at 0.8 microliter each were made at approximately the rostral and caudal margins of the injury with the aim to bridge the lesion. Equal volumes of saline were injected in control animals. With this procedure, no swelling of the spinal cord was observed, and the dorsal spinal artery was not affected. On average $1.4 \times 10^{5} \pm$ $2.3 \times 10^{4}$ cells per mouse were grafted. Subsequently, the wound was closed with suture and wound clips (Fine Science Tools Inc., Foster City, CA). The mice were placed on a warming pad until they were alert and upright.

\section{Spinal Evoked Potential (SpSEP) Measurements}

SpSEPs were recorded from 19 spinal cord injured (SCI) mice treated with EPI-NSC, 22 SCI mice treated with saline and 6 uninjured mice 4 months following injury and treatment.

The technique used to evoke SpSEPs has been described previously [17]. Briefly, animals were anesthetized (as above), shaved and securely taped to a Styrofoam surgical board. For recording, two Teflon insulated 7-strand, $0.0254 \mathrm{~mm}$ diameter, stainless steel wires with a $3 \mathrm{~mm}$ region of deinsulation (Fig. 1) were placed subcutaneously at the level of T4, rostral to the site of injury. The recording electrodes were connected to a high input impedance module with an input impedance of $2 \mathrm{~T} \Omega$ and capacitance of $10 \mathrm{pF}$ (Model HZP; Grass-Telefactor, West Warwick, $\mathrm{RI}$ ), which, in turn, was connected to an amplifier (Model QP511 Quad AC Amplifier System; Grass-Telefactor, West Warwick, RI). The amplifier was connected to a laptop computer via a data acquisition board.

For stimulation, a $23 \mathrm{G}$ needle (cathode) was placed subcutaneously at the midline, $3 \mathrm{~cm}$ caudal to the most caudal recording electrode. A blunt tipped, $23 \mathrm{G}$ hypodermic needle served as the anode. The anode was placed in a position to stimulate the tibial nerve through the skin at the 


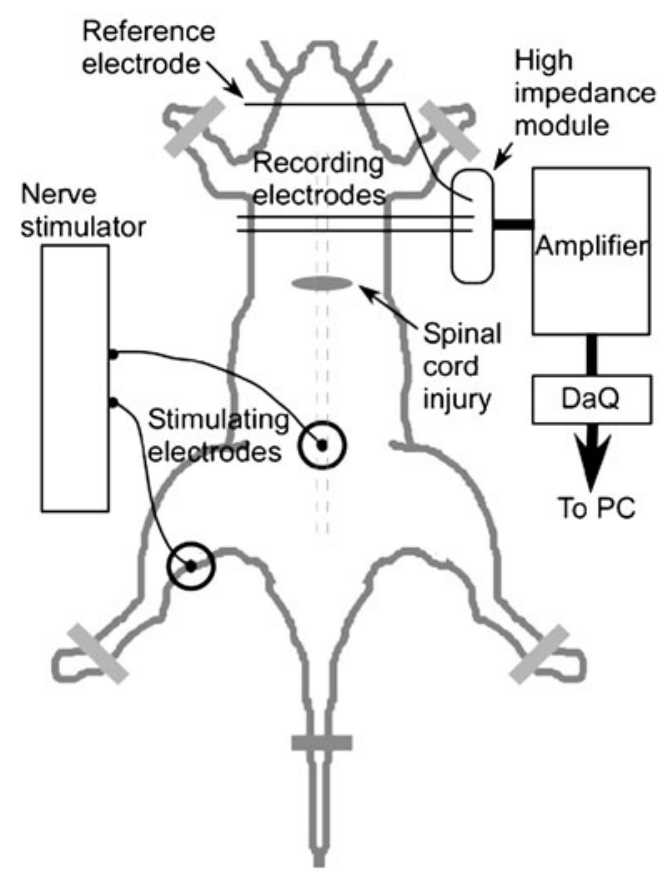

Fig. 1 Experimental setup for collecting spinal somatosensory evoked potentials (SpSEPs). Teflon insulated stainless steel fine wire electrode used for recording SpSEPs. The electrodes were positioned subcutaneously above the vertebral body and paraspinal muscles. The recording electrodes were connected to a high impedance module, amplifier, and data acquisition system. Stimulating electrodes delivered electrical stimulation to the tibial nerve through the skin over the popliteal fossa

back of the knee (over the popliteal fossa). Placement of the anode was adjusted to elicit plantar flexion of the hind paw with a minimum stimulus current.

Somatosensory stimuli to the tibial nerve were delivered by a constant current nerve stimulator (Model DS7A; Digitimeter, Hertfordshire, UK). The stimulation frequency was $3.5 \mathrm{~Hz}$ and the pulse duration was $500 \mu \mathrm{s}$. Electrical stimulation was delivered at three stimulus intensities: $1 \mathrm{X}$, $2 \mathrm{X}$ and $3 \mathrm{X}$ the threshold current for motor response. Motor threshold current was between 0.47 and $0.49 \mathrm{~mA}$ for all animals and was similar across groups. Separate SpSEP recordings were done for each leg and each stimulus intensity.

Continuous data were segregated into epochs; each epoch was defined as the voltage recorded between -20 and $150 \mathrm{~ms}$ around the time of stimulus $(0 \mathrm{~ms})$. Each epoch was baseline corrected and 700 epochs were ensemble averaged for each recording to obtain the SpSEP waveform. The amplitude of the mid- to long-latency component of the SpSEP was defined as the range of signal amplitude (maximum amplitude-minimum amplitude) between 8 and $25 \mathrm{~ms}$ after the stimulus $[17,18]$.

A two-way analysis of variance (ANOVA) with post-hoc Tukey's test was performed to compare the SpSEP amplitudes across groups and limbs at each stimulus intensity. The peak-to-peak amplitude of the mid latency SpSEP compo- nent was taken as the dependent variable, with two fixed factors: (i) the side of stimulation (left and right leg) and (ii) the type of treatment (uninjured, EPI-NCSC injected and saline injected mice). Threshold for statistical significance was fixed at $p<0.05$ and SPSS 16.0 statistical software (SPSS inc. Chicago, IL) was used for analysis.

\section{Semmes-Weinstein Touch Test}

Cutaneous sensation was evaluated using the SemmesWeinstein test according to manufacturer's instructions. The Touch-Test ${ }^{\mathrm{TM}}$ Sensory Evaluators (North Coast Medical Inc, Morgan Hill, CA) consist of an array of filaments of different thicknesses corresponding to specific weights ranging from 0.008 to $300 \mathrm{~g}$. The filament tip was aimed at the middle of the paw and pressed at a $90^{\circ} \mathrm{C}$ angle against the skin and held in place for 1.5-2 s. A clasping reflex, when it occurred, was immediate, as was clonus. Both ceased when the filament was removed. Clasping was considered indicative of a response. Limbs were tested multiple times and responses occurred reproducibly. In order to avoid desensitization, there was a 2 min waiting period between repeat tests. Threshold weight values for the clasping reflex were used for statistical analysis. Data were analyzed by ANOVA and post-hoc comparisons as described for the SpSEP measurements.

\section{Immuno-electron Microscopy}

For immuno-electron microscopy spinal cords were fixed in situ in order to preserve ultrastructure of the spinal cord tissue as much as possible. Mice were deeply anesthetized, the spinal cord exposed and transected immediately rostral and caudal of the lesion/graft. Several drops of $4 \%$ paraformaldehyde (PFA) were placed at the level of the cuts and on top of the spinal cord. After $10 \mathrm{~min}$, the mice were euthanized by anesthetic overdose. The pertinent section of spinal cord was removed, cut into smaller pieces and further incubated in 4\% PFA in $0.1 \mathrm{M}$ phosphate buffer at $\mathrm{pH} 7.3$ for $30 \mathrm{~min}$. The tissue pieces were then inspected with the fluorescence microscope for EGFP signal. Fluorescent pieces of tissue were selected and further trimmed to approximately $200 \mu \mathrm{m}$ sized pieces in order to fit them into a cryo planchette from a Leica EMPact II high pressure freezing apparatus. The samples were high pressure frozen and freeze substituted in ethanol containing $0.5 \%$ uranyl acetate into Lowicryl $\mathrm{K} 11 \mathrm{M}$ resin following the protocol of Ameye et al [19]. Ultrathin sections were cut and mounted on formvar-coated copper grids and sections probed with anti-GFP polyclonal rabbit serum (MBL Co Ltd, Naka-ku Nagoya, Japan). AntiGFP antibody was visualized using goat anti-rabbit $10 \mathrm{~nm}$ gold probe (EMS, Hatfield, PA, USA) and viewed on a Hitachi H600 transmission electron-microscope. 


\section{Retrograde Tracing}

Mice were anesthetized by intraperitoneal injection of Domitor $(1 \mathrm{mg} / \mathrm{kg}$, Pfizer Animal Health, New York, NY 10017) and Ketamine (75 mg/kg, BEDFORD Laboratories, Bedford, OH 44146). A laminectomy was performed at thoracic level 12. A sharpened tungsten needle the tip of which had been coated with a concentrated solution of Alexa Fluor 680-conjugated dextran (10,000 MW; Molecular Probes/ Invitrogen, Carlsbad, California 92008 USA) and subsequently dried [20] was placed on the left side of the spinal cord, the dura punctured and the needle carefully pushed $1 \mathrm{~mm}$ deep into the tissue. The wound was closed with suture and wound clips (Fine Science Tools (USA), Inc. Foster City, CA). The mice were placed on a warm pad until they were awake and upright. After one week, animals were anesthetized as described above and perfused intracardially with $0.9 \%$ saline followed by a solution of $4 \%$ PFA in 0.1 molar phosphate buffer ( $\mathrm{pH}$ 7.4). After perfusion, the spinal cord was removed and postfixed in a fresh solution of the same fixative at $4^{\circ} \mathrm{C}$ for over $24 \mathrm{~h}$, and subsequently cryoprotected and sectioned at 12 micrometer.

\section{Real Time Polymerase Chain Reaction (qPCR)}

For reverse transcription, total RNA was extracted with TRIzol reagent (Invitrogen) and treated with DNaseI (Invitrogen). First strand cDNA was synthesized using the SuperScript III First Strand synthesis kit from Invitrogen, with Oligo (dT) used as the primer according to manufacturer's instructions. For quantitative PCR (qPCR), $10 \mu 1$ reactions consisted of $5 \mu 12 \times \mathrm{RT}^{2}$ Real-Time SYBR Green/ ROX MasterMix (SABiosciences/Tebu-Bio), $0.5 \mu$ l First Strand cDNA template and $0.5 \mu 1 \mathrm{RT}^{2}$ qPCR Primer Set (SABiosciences/Tebu-Bio) and was brought to a $10 \mu \mathrm{l}$ final volume with $\mathrm{ddH}_{2} \mathrm{O}$. Thermo-cycling conditions were as follows: $95^{\circ} \mathrm{C}, 10 \mathrm{~min}$ and 40 cycles of $95^{\circ} \mathrm{C}, 15 \mathrm{~s}, 60^{\circ} \mathrm{C}$ $60 \mathrm{~s}$ and $80^{\circ} \mathrm{C} 30 \mathrm{~s}$. Melting curve analysis showed a single amplification peak for each reaction. Ct values for targets were expressed as relative expression compared to the average of three housekeeping genes (GAPDH, $\beta$-actin and Ribosomal Protein S9).

\section{Results}

\section{Ex Vivo Expansion of EPI-NCSC}

All cells in primary explants were EGFP-positive (Fig. 2). For sub-culturing, the bulge explants were removed, leaving the EPI-NCSC in the culture plates. The cells were subsequently detached by trypsin treatment and placed on a slurry of hydrated sterile collagen microcarriers (50 mg dry weight; gift of Titan Pharmaceuticals, South San Francisco, CA) in a $35 \mathrm{~mm}$ plastic Petri dish (Greiner Bio-One North America Inc., Monroe, NC 28110) in $1.5 \mathrm{ml}$ expansion medium. Cells attached to the surface of the microcarriers within 30-60 min and continued to divide rapidly (Fig. 3a, b). The culture medium was exchanged every other day, and then daily towards the end of the 9-10 day expansion period in secondary culture. For medium changes, the entire culture was transferred to a $15 \mathrm{ml}$ centrifuge tube, the carriers allowed to settle for 1-2 min, the supernatant medium removed, replaced with an equal volume of fresh medium and the culture then transferred back to a new $35 \mathrm{~mm}$ Petri dish.

The expansion medium consists of Neurobasal-A (without Vitamin A; Invitrogen, Carlsbad, CA); 10\% chick embryo extract; $2 \%$ fetal bovine serum (Hyclone, Waltham, MA), B27 supplement (GIBCO/Invitrogen, Carlsbad, CA), ITS + 3 (insulin, transferrin selenium plus three essential fatty acids; Sigma/Aldridge, St. Louis, MO), GlutaMAX (Invitrogen), stem cell factor ( $10 \mathrm{ng} / \mathrm{ml}$; R\&D Systems, Minneapolis, MN), neurotrophin 3 ( $20 \mathrm{ng} / \mathrm{ml}$; Promega, Madison, WI), fibroblast growth factor-2 (50 ng/ml; Upstate, Jaffrey, $\mathrm{NH})$ and epidermal growth factor (25 ng/ml; Sigma/Aldrich). During ex vivo expansion key stemness genes (Myc, Klf2, Lin28, Sox 2 and Sox 10) remained expressed at levels similar to those of embryonic stem cells (ESC) as determined by real-time PCR (Fig. 3c). By contrast, Nanog and Oct-4 were 700-800 fold lower than in mouse embryonic stem cells (Fig. 3c). Thus transcript levels were virtually identical to those we have determined for unexpanded cells in primary explants [21]. Typically we obtained 2 million expanded stem cells from 15-20 bulge explants within 10-12 days.

\section{Bilateral Improvement as Measured by Spinal Somatosensory Evoked Potentials}

A mid latency component of the SpSEP was observed in all animals as a biphasic deflection occurring between 8 and $25 \mathrm{~ms}$ after the stimulus (Fig. 4a). This component reflects the spinal cord conduction of myelinated A-fiber sensory input to the lateral spinothalamic tract [22]. The short latency SpSEP components (occurring around $5 \mathrm{~ms}$ post stimulus) were obscured by the stimulus artifact in most animals due to the extremely short conduction time between the stimulating and recording electrodes in mice [17]. Additionally, the long latency components, evoked by C fiber input to the lateral spinothalamic tracts $[23,24]$ were not observed in all animals, possibly because of the low stimulus intensity used [17].

Analyses were performed at 4 months post-injury/stem cell graft. Electrical stimulation of the left and right tibial nerves were performed and SpSEP responses assessed. In uninjured mice the average SpSEP response was $29.9 \pm$ 5.1 microvolts and $29.08 \pm 7.3$ microvolts upon stimulation 
Fig. 2 Bulge explant from whisker follicle. Bulge explants were microdissected from whisker follicles of C57BL/6-TgN (ACTbEGFP)1Osb mice, in which virtually all cells express EGFP. a, green fluorescence; $A$, phase contrast optics; the explant is surrounded by a halo of emigrating cells with stellate morphology. $\mathbf{b}, B^{\prime}$; boxed area in A1 at higher magnification. All EPI-NCSC are green fluorescent
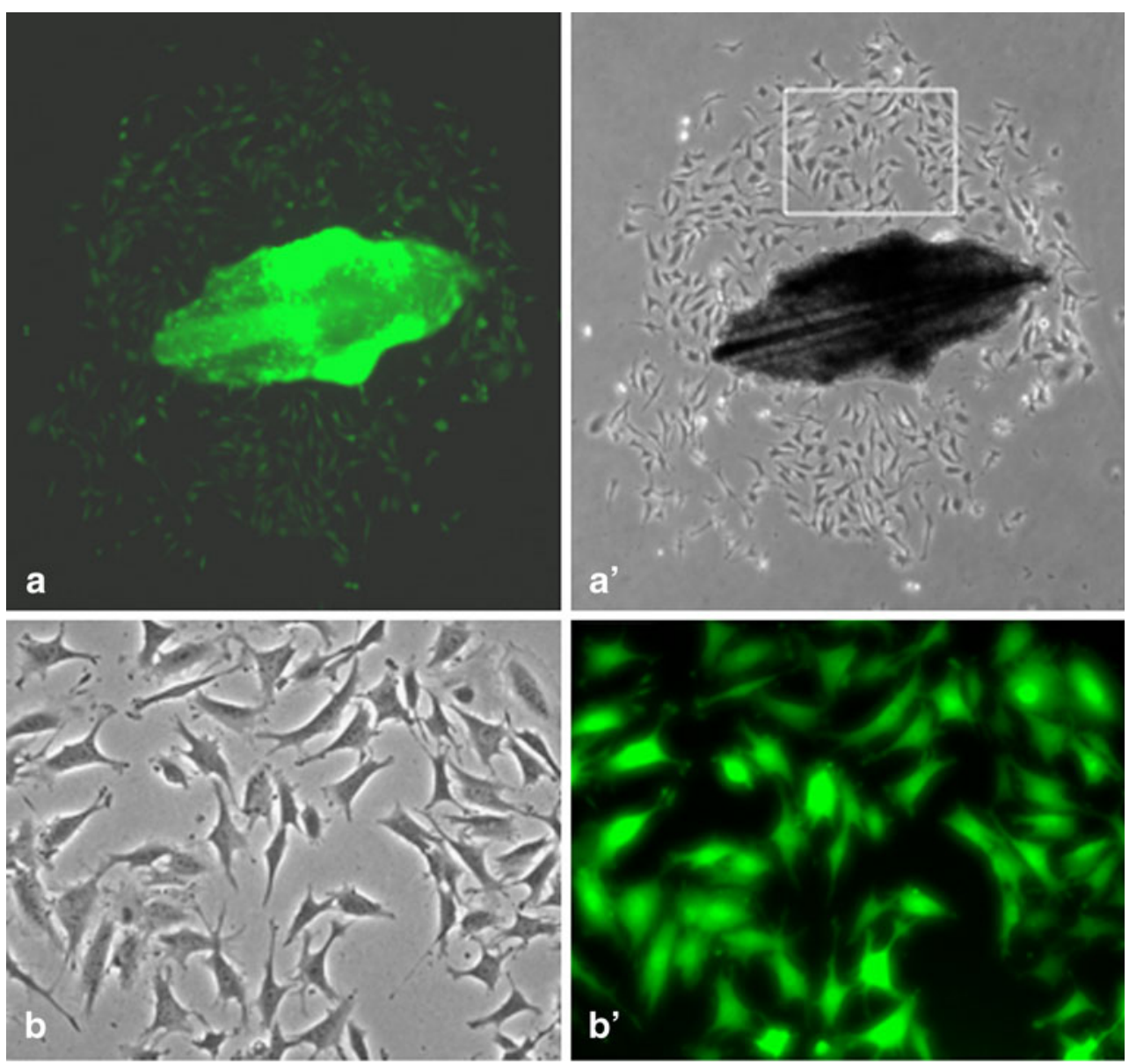

of the sciatic nerve in the left and right hind leg, respectively (Fig. 4b). By contrast, in negative control mice that received a midline contusion and saline injection instead of stem cells, the average SpSEP response dropped to $6.05 \pm 2.1$ and $6.39 \pm 2.0$ microvolts on the left and right sides, respectively (average of 20 mice; $p<0.01$ ). In mice that received a midline contusion lesion and EPI-NCSC grafts on the left side of the spinal cord, average SpSEP response was $13.27 \pm 3.1$ on the left side and $12.86 \pm 3.5$ on the right side, respectively (Fig. 4b). Notably, percent changes were similar on the left and right hind legs. In summary, the injury reduced SpSEPs to $20 \%$ of uninjured mice, whereas in mice with a midline contusion and stem cell implant on the left side of the spinal cord SpSEP response was $44.4 \%$ of uninjured mice, which represents a greater than $24 \%$ bilateral improvement.

Bilateral Improvement as Measured by Semmes-Weinstein Touch Test

In parallel with SpSEP measurements, we performed the Semmes-Weinstein touch test on mice from the same cohort 4 months after injury/graft. When there was no response even at $300 \mathrm{~g}$, the value of $300 \mathrm{~g}$ was used nevertheless. In saline control mice, three of four left hind legs and three of four right hind legs did not show any response at $300 \mathrm{~g}$. The results for saline control mice thus represent an underestimate. On average, the threshold response on the left hind leg was $7.7 \pm 0.9 \mathrm{~g}$ in non-injured mice, $242.2 \pm 29.9$ in saline controls, and $5.2 \pm 0.5$ in mice with an EPI-NCSC graft (Table $1 ; p=$ $0.0001)$. Stimulation of the paw of the right hind leg resulted in similar responses; $7.3 \pm 0.8$ (non-injured), $141.5 \pm 50.2$ (saline), and 5.2 \pm 0.5 (EPI-NCSC graft) grams (Table 1; $p=$ $0.0001)$. Thus despite having grafted the stem cells in the left side of the spinal cord only, recovery for touch perception was bilateral, similar to the observations with SpSEP measurements.

In some mice a clonus was observed in the hind limbs at weights of $0.6-1.4 \mathrm{~g}$, but not at higher probe weights. No clonus presented in the forelimbs of experimental and control groups. The lesion also affected the left and right forelegs. The deficit was more pronounced in the left forelimb (Table 1) and improved statistically significantly over saline control in the presence of EPI-NCSC grafts (Table 1; line 3, columns 1 and 2). These observations indicate that the lesion at $\mathrm{T} 8$ caused deficiencies at more rostral levels also, and that these were ameliorated on the side of transplantation in the presence of EPI-NCSC grafts, 

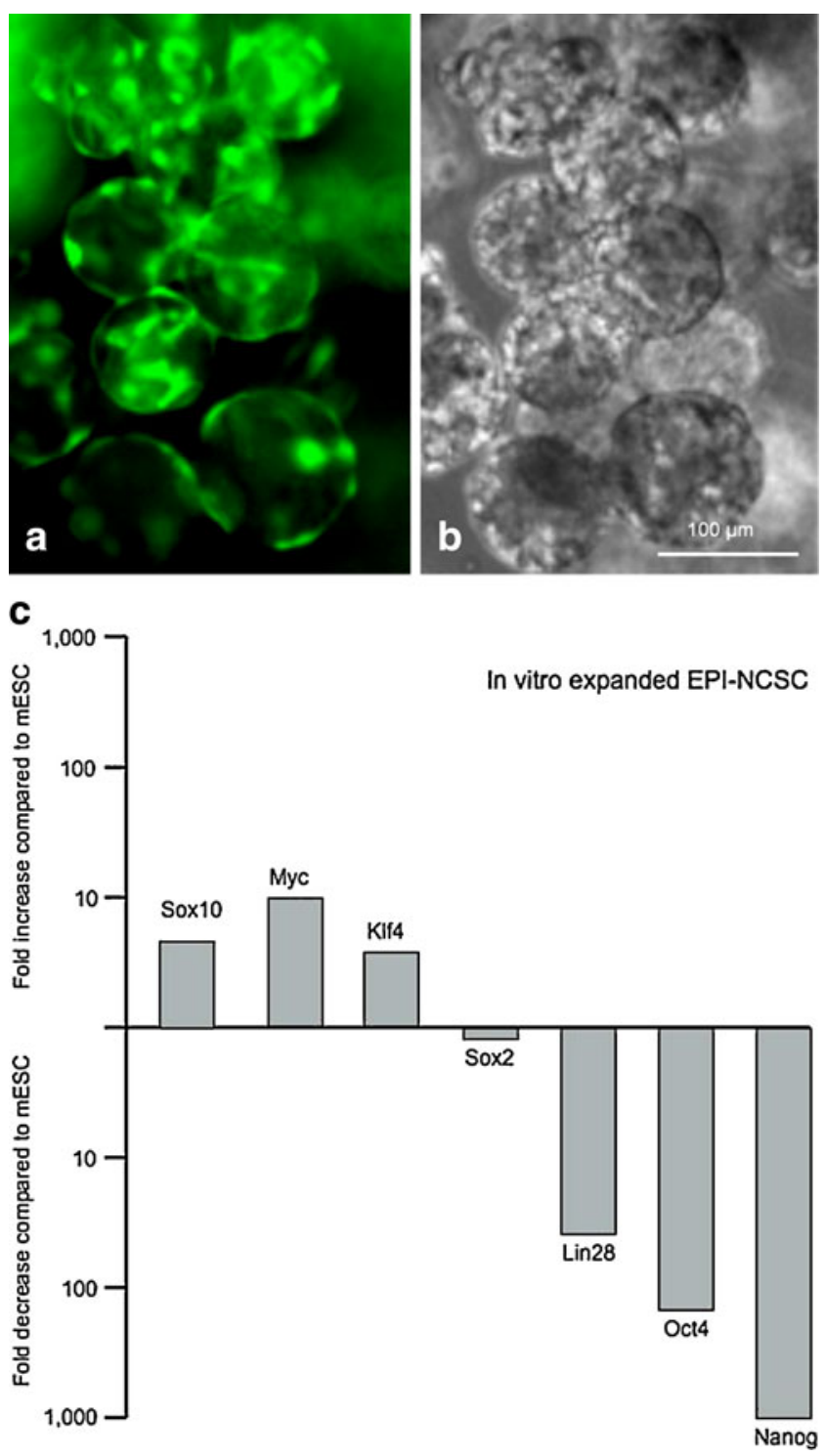

but not, or to a lesser degree, on the contralateral side or in saline controls. We tested for allodynia, both in the paws and at the area of surgery. We did not observe retraction of limbs in any of the mice or any obvious discomfort when probed at the site of surgery. We thus conclude that at 4 months post-surgery allodynia was not present at detectable levels. Overall, similar to the SpSEP data, the Semmes-Weinstein touch test indicates a bilateral improvement of sensory perception in the absence of allodynia.

\section{Differentiation of Grafted EPI-NCSC into Myelinating Oligodendrocytes}

As we have shown previously, subsets of grafted EPINCSC expressed oligodendrocyte markers, but not markers for astrocytes or Schwann cells [4]. Here we show by immuno-electron microscopy that grafted EPI-NCSC can differentiate into myelinating oligodendrocytes (Fig. 5).
Fig. 3 In vitro expansion of EPI-NCSC. (a, b) During in vitro expansion, EPI-NCSC were cultured on collagen microcarriers. $(A)$ Enhanced green fluorescent protein fluorescence of cells growing on microcarriers. Cells adhered to the surface of the microcarrier, divided and migrated. At high cell densities, cells started to populate neighboring microcarriers. $(B)$, phase contrast optics of the same field as in $(A)$. The microcarriers are visible. (c) Validation of expression of key stemness genes after expansion. Data were obtained by qPCR and are expressed as fold-increase/decrease compared to expression levels in mouse embryonic stem cells. The expression levels for all seven genes are virtually the same as we have published for EPI-NCSC from primary explants prior to expansion [21]. This result thus indicates that stemness does not change significantly during ex vivo expansion of mouse EPI-NCSC. Sox10, a neural crest stem cell marker, and Myc are 8-fold and 10-fold higher expressed in EPI-NCSC than in mouse ES cells, respectively. Klf4 and Sox 2 are expressed at levels comparable to those in embryonic stem cells, whereas Lin28 is expressed at 80-fold lower levels than in ES cells. Like in nonexpanded EPI-NCSC Oct-4 and Nanog are expressed at several hundred to thousand fold lower levels than in ES cells, which might explain the observation that EPI-NCSC do not form tumors in the mouse spinal cord

Grafted cells were able to myelinate processes elaborated by other grafted cells (Fig. 5A', c), as well as processes of putative host cells (Fig. 5b,c). Thus at the subcellular level, it became evident that subsets of intraspinal EPI-NCSC can differentiate into functional oligodendrocytes.

\section{Retrograde Transport}

Tracing of processes by retrograde transport of Alexaconjugated dextran showed that red fluorescent retrogradely transporting neurites and grafted cells can intermingle (Fig. 6a$\mathrm{A}^{\prime}$ ), suggesting that at least some host neurites are able to traverse the scar. Not only host neurites, but also a subset of EPI-NCSC-derived green fluorescent neurites and cell bodies showed red fluorescence (e.g., marked area in Fig. 6b-B", arrowhead). There were also green fluorescent grafted cells that had not internalized the dye (Fig. 1b-B", inset).

\section{Absence of Grafted EPI-NCSC on the Right Side} of the Spinal Cord and Absence of Processes in the Dorsal Root

Next we looked for explanations for the bilateral improvement in sensory perception in the presence of unilateral stem cell grafts. Intraspinal EPI-NCSC did not migrate laterally to the right side of the spinal cord (Fig. 7). In addition we inspected cross-sections at the level of graft (Fig. 8a-A"') and up to three segments below and above the lesion. Figure 8b-B"' show a section three levels above the lesion. In none of the sections did we observe EGFPfluorescent cells or processes in the dorsal/ventral roots or in the dorsal root ganglion. Together, the data show that bilateral improvement was not due to cell migration and therefore points to production of diffusible factors by intraspinal EPI-NCSC. 
Fig. 4 Somatosensory evoked potentials. (a) Typical SpSEP response from representative mice from EPI-NCSC treated group (study group, black trace) and saline treated group (control group, grey trace) and uninjured group (stippled trace). The mid latency component is much larger in the EPI-NCSC treated mouse. A short latency component is also seen abutting the stimulus artifact in the SpSEP response obtained from the EPINCSC treated mouse. However, the short latency component was obscured by the stimulus artifact in most recordings. (b) Mean amplitude of the mid-latency SpSEP response across groups and stimulation side (right or left hind limb). Mid-latency SpSEP amplitude from age matched uninjured mice is also shown for comparison
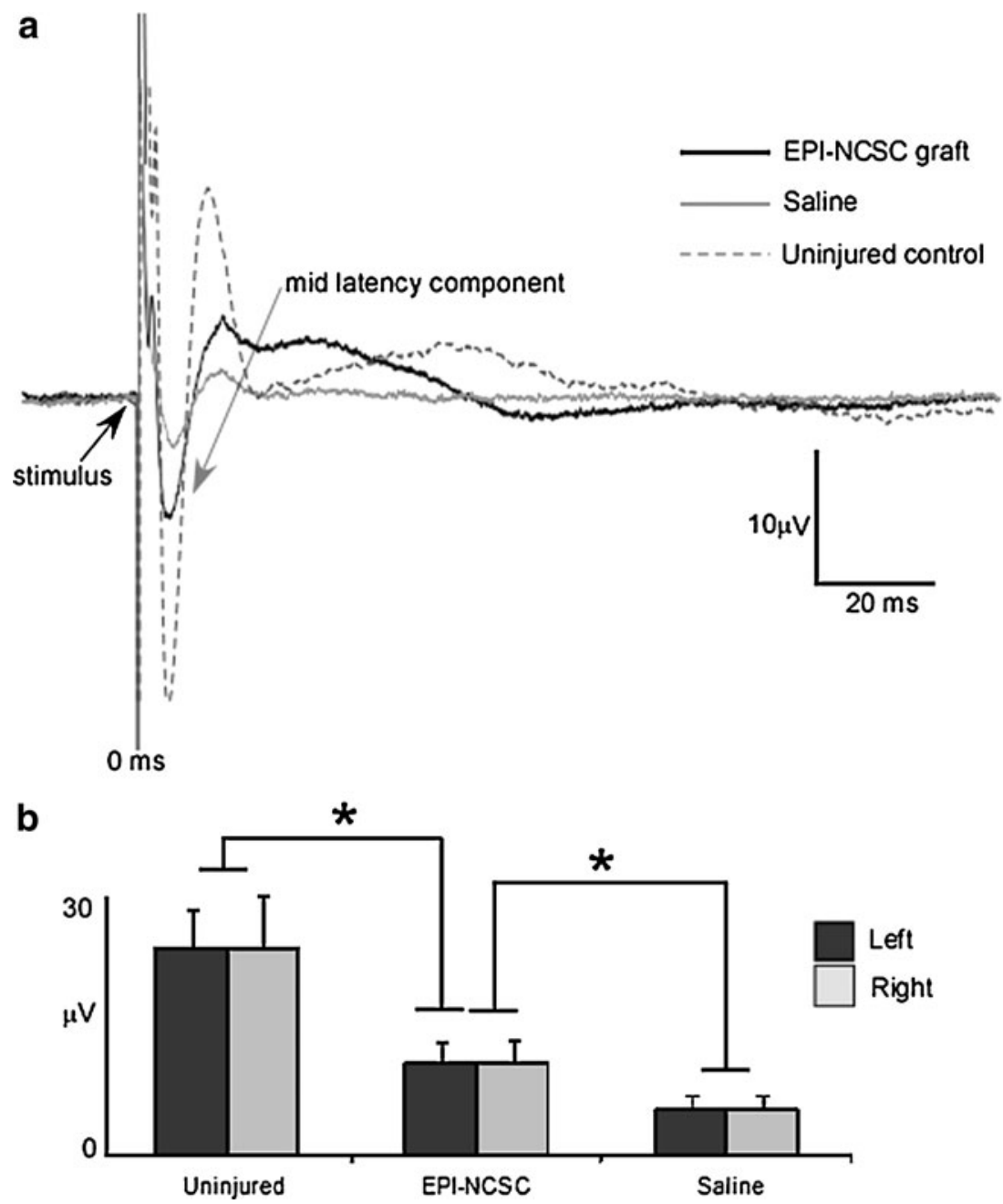

Expression of Neurotrophins, Angiogenic Factors and Matrix-Degrading Enzymes by EPI-NCSC

One explanation for the bilateral improvement of sensory perception observed in our present study could be the

Table 1 Semmes-Weinstein touch test

\begin{tabular}{lccc}
\hline Limb tested & \multicolumn{3}{c}{ Threshold response \pm SEM (gram) } \\
\cline { 2 - 4 } & EPI-NCSC graft & Saline control & Non-injured \\
\hline Left hind leg & $5.2 \pm 0.5^{\mathrm{a}}$ & $242.2 \pm 29.9^{\mathrm{ab}}$ & $7.7 \pm 0.9^{\mathrm{b}}$ \\
Right hind leg & $5.2 \pm 0.5^{\mathrm{c}}$ & $141.5 \pm 50.2^{\mathrm{cd}}$ & $7.3 \pm 0.8^{\mathrm{d}}$ \\
Left front leg & $1.2 \pm 0.3^{\mathrm{e}}$ & $2.7 \pm 0.8^{\mathrm{e}}$ & $1.8 \pm 0.5$ \\
Right front leg & $1.1 \pm 0.2$ & $2.3 \pm 0.7$ & $1.4 \pm 0.2$ \\
\hline
\end{tabular}

a, b, c, d $p=0.0001$

${ }^{\mathrm{e}} p=0.04$

EPI-NCSC graft; $n=4$

Saline Control; $n=5$

Non-injure mice; n-6 expression of growth factors by EPI-NCSC, which could diffuse and thus affect the host environment bilaterally. We here present evidence for the expression of three classes of relevant factors, (1) neurotrophins and other growth factors, (2) angiogenic factors and (3) metalloproteases and other extracellular proteases capable of modifying scar formation.

EPI-NCSC express numerous neurotrophic factors, including for instance nerve growth factor, brain-derived neurotrophic factor, fibroblast growth factors, insulin-like growth factor 1 and bone morphogenetic proteins (Table 2). EPI-NCSC also express the angiogenic factors Vegfa and Vegfb (Table 2).

Extracellular proteases are involved in the degradation of extracellular matrix and release of membrane-bound growth factors. In the spinal cord they have thus been applied to modify scar formation (see e.g., [11]) and activation of growth factors. EPI-NCSC express many metalloproteases and other extracellular proteases, which break down extracellular matrix. Mmp-2 breaks down denatured collagen. Mmp-3 breaks down proteoglycans, fibronectin, laminin, and collagens III, IV, IX, and X, whereas Mmp- 

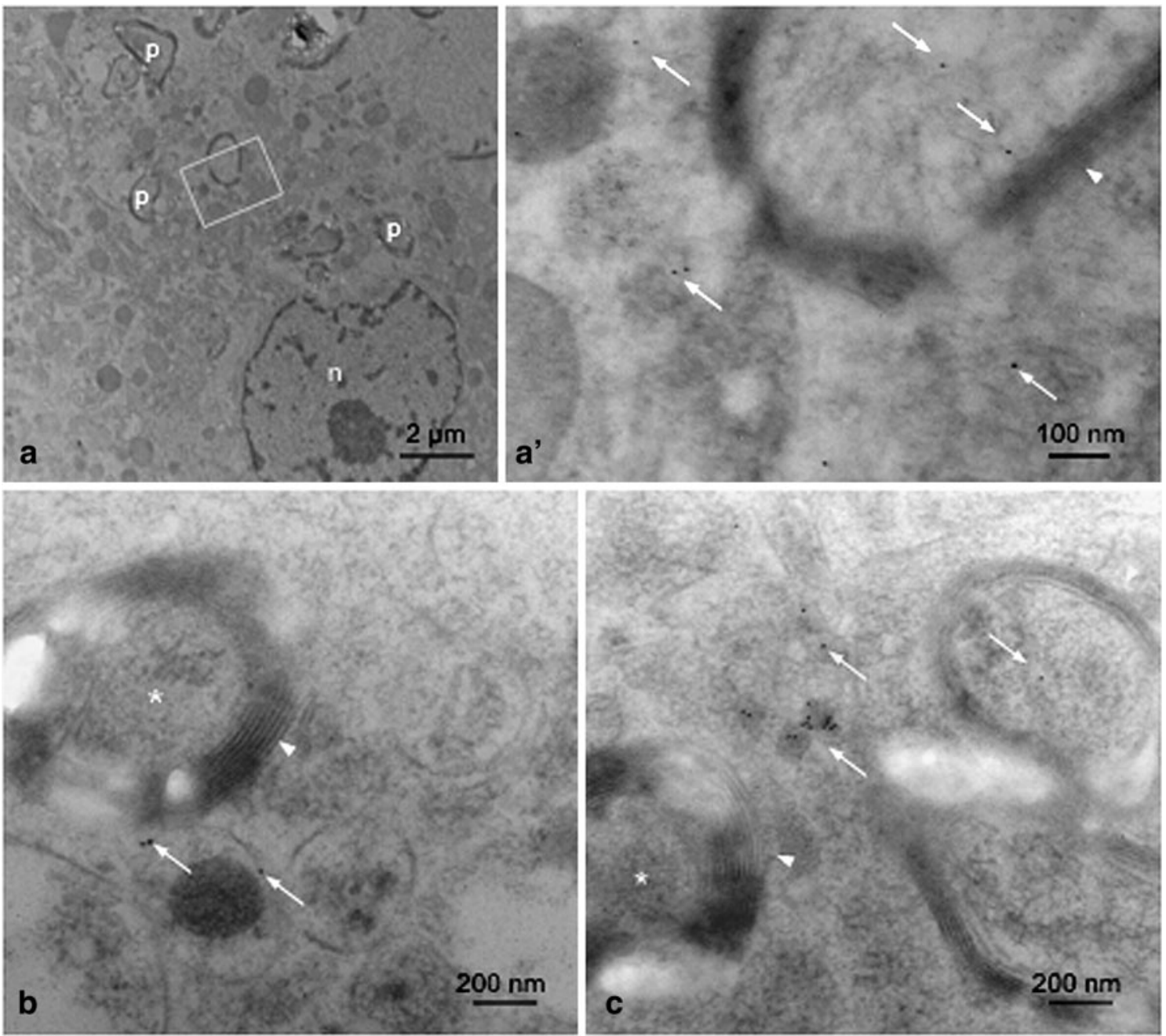

Fig. 5 Differentiation of intraspinal EPI-NCSC into myelinating oligodendrocytes. Immuno-electron microscopy showed that instraspinal EPI-NCSC can differentiate into myelinating oligodendrocytes. a) low magnification of a cell showing the nucleus (n) and several myelinated processes (p). A'; higher magnification of are boxed in A. Several gold particles (e.g., arrows) are visible identifying the cells as a transplanted EPI-NCSC-derived cell. Part of a myelinated (arrow-

14 activates Mmp-2. ADAMts-1 degrades extracellular matrix and pro-collagens (Table 2).

\section{Discussion}

In this study we showed that unilateral EPI-NCSC transplants into midline spinal cord lesions caused bilateral improvement in sensory connectivity and touch perception. EPI-NCSC grafts elicited a $24 \%$ bilateral improvement in sensory perception, as measured by SpSEP responses. Touch perception was significantly improved also, as determined by the Semmes Weinstein test, and there was no allodynia. Furthermore, we have developed a protocol for the ex vivo expansion of mouse EPI-NCSC into

head) process is visible. The process also contains gold particles (e.g., arrows), identifying it as a EPI-NCSC-derived process. b; another EPI-NCSC-derived cell (arrows) has myelinated (arrowhead) a process that does not contain gold particles (asterisk), and thus could be of host origin. c; a third cell of EPI-NCSC origin (arrows) that envelops processes of graft origin (arrow) and of presumably host origin (e.g., asterisk) level

millions of stem cells. We have also provided evidence that subsets of grafted cells can acquire properties of functional neurons, i.e. by SpSEP response and retrograde transport, and that others differentiate into myelinating glia. The latter are presumably oligodendrocytes, as we have shown previously that intraspinal EPI-NCSC do not express markers for astrocytes and Schwann cells, but express epitopes specific to oligodendrocytes [4]. Finally, we provide evidence that EPI-NCSC express multiple genes that encode neurotrophic factors, angiogenic factors and metalloproteases, the sum of which may explain the observed bilateral functional improvements.

For the design of the expansion medium we relied on our longSAGE gene expression profile, which revealed the expression of relevant growth factor receptors [15]. The 

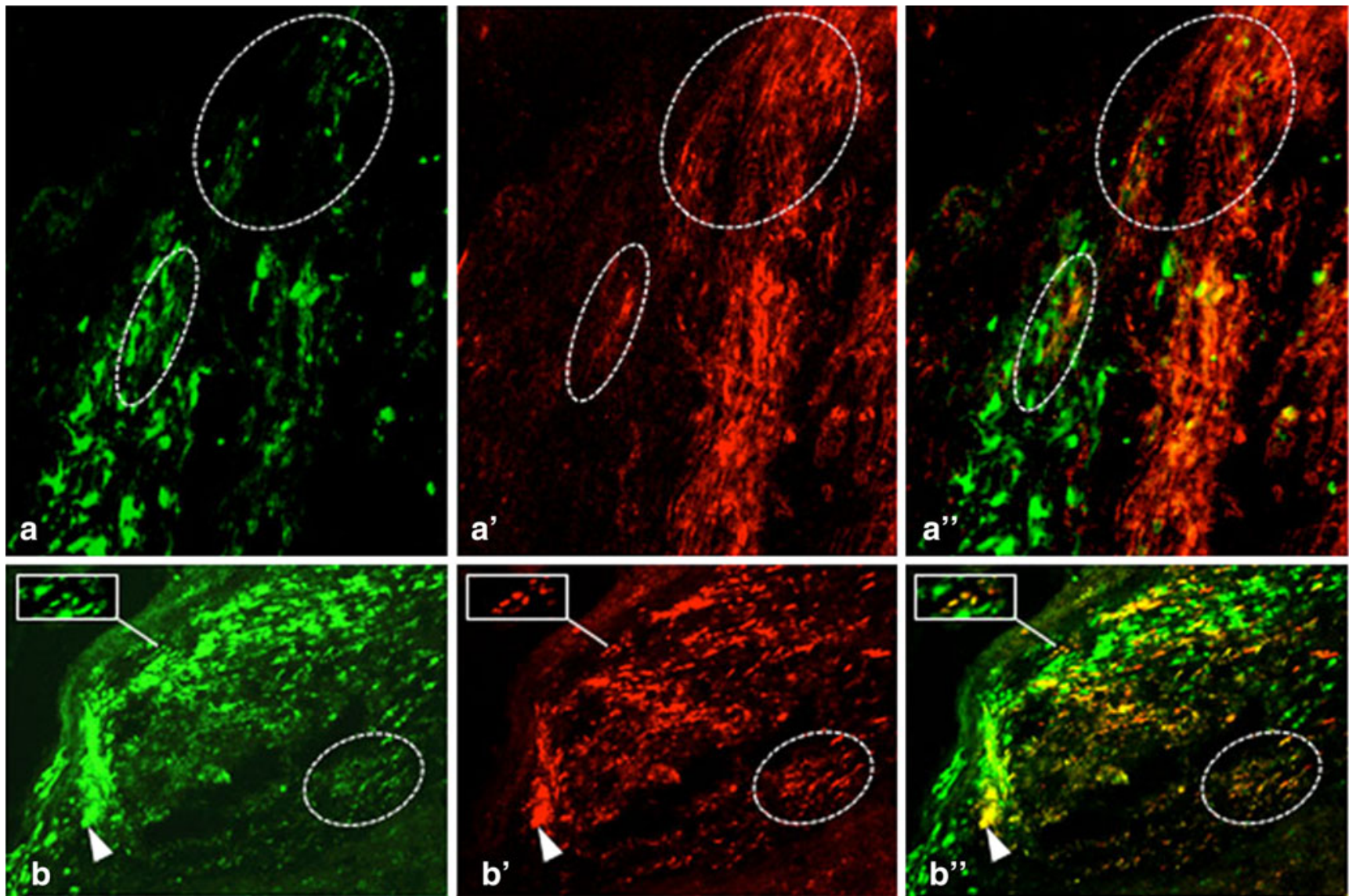

Fig. 6 Retrograde transport. EPI-NCSC were injected at T8. The retrograde tracer was injected 4 segments below, at T12. a $-\boldsymbol{A}^{\prime \prime}$. Image at level of graft. a, green fluorescence illumination showing and area containing grafted cells. A', same area as in a in far-red fluorescence illumination. Many host fibers are visible. Several areas are visible where host fibers traverse areas of grafted cells (e.g., circled areas). A'; merged images of a and A'. $\mathbf{b}-\mathbf{B}$ '. Image at level of graft that show EPI-NCSC-derived somata and fibers. $\mathbf{b}$; Clusters of EPI-NCSC (b, arrowhead) and cross sections of EPI-NCSC-derived fibers are present (e.g., circled area). B'; same area as in $\mathbf{b}$ showing far-red fluorescence of the retrograde tracer. $B$ "; Merged images $\mathbf{b}$ and $B$ '. Yellow areas indicate co-localization. A subset of cell bodies have taken up and presumably transported the dye (e.g., arrowhead). Many green-fluorescent processes are also red-fluorescent (e.g., circled area). The inset shows a higher magnification of a mixture of crosssectioned fibers, some of which do not contain the retrograde tracer, whereas others are both green and red fluorescent as indicated by yellow color. From retrograde tracing experiments we can conclude that (1) host fibers can traverse the graft; (2) some graft-derived cells can internalize and retrogradely transport the tracer, indicating that their processes can reach up to four segments below the site of grafting and (3) that they show an aspect of functionality by being able of retrograde transporting

reduces costs for growth factors. Since EPI-NCSC are migratory and have a tendency to avoid each other, they were able to populate adjacent microcarriers. Therefore sub-culture was not necessary, but new microcarriers could be added instead. An additional benefit of microcarrier culture is that occasional dead cells detach and therefore are removed with the supernatant during culture medium exchanges. Finally, at the end of the culture period, the stem cells can be isolated gently and efficiently by dissolving the microcarriers with collagenase, which leads to populations of virtually $100 \%$ viable and single cells.

An important aspect of the current study was improvement of sensory connectivity and touch perception. We show that intraspinal EPI-NCSC also did not migrate laterally, nor did they invade the spinal roots or the dorsal 


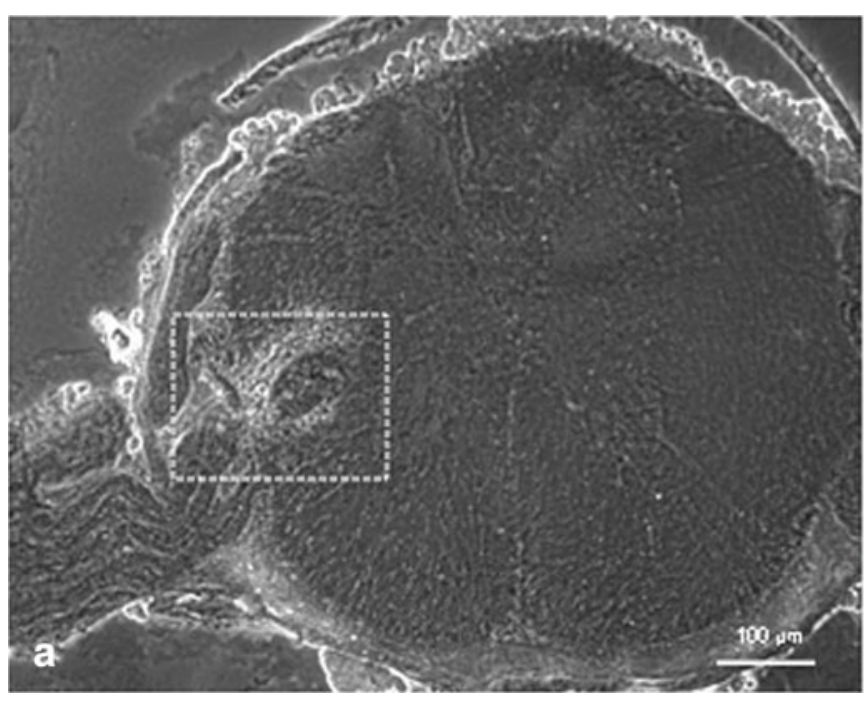

Fig. 7 Location of graft. Cross sections of the spinal cord at the level of the injury and graft showed that the EPI-NCSC graft remained on the left side of the spinal cord. No EPI-NCSC were observed in other locations. Importantly they were not observed on the right side of the spinal cord. a, phase contrast optics; $\mathbf{b}$, boxed area in a at higher

root ganglia. We thus conclude that a likely mechanism for functional improvement on both sides of the spinal cord is the production, release and diffusion of trophic factors, angiogenic factors and/or enzymes that can modify scar formation. This notion is supported by our real-time PCR data presented in Table 2. It can be envisioned that EPINCSC derived growth factors diffuse from the graft and

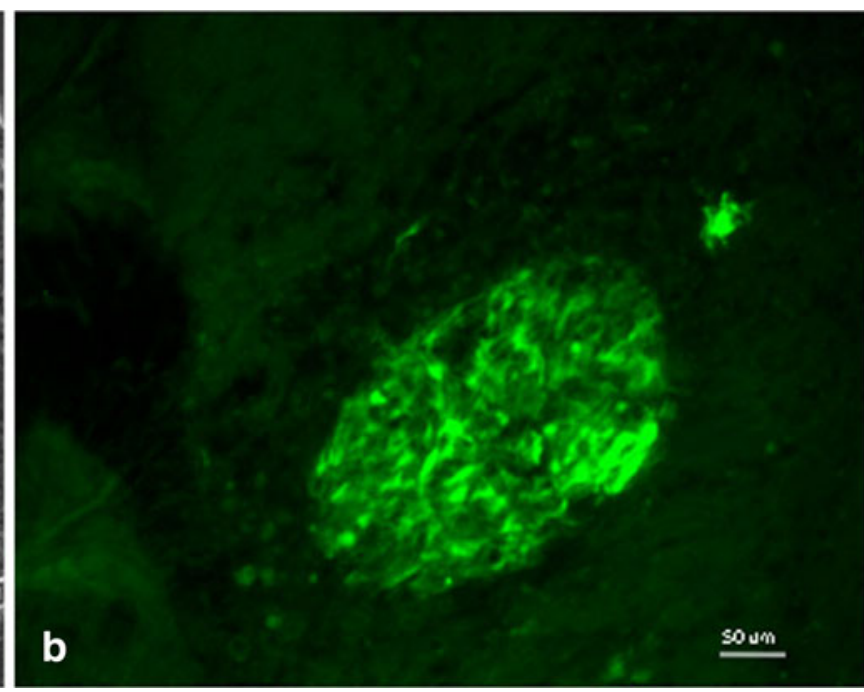

magnification and under fluorescence illumination. Green fluorescent cells are grafted cells. They are localized at the site of injection. Since grafts were not globular, a cluster of cells can be seen in this section that seems to be disconnected from the main graft

thus can have a bilateral protective and/or regenerative effects on the host tissue. By contrast, or in addition, they could also serve to activate endogenous regeneration mechanisms, as has been suggested for instance by Hooshmand et al. [27]. The injury spread rostrally. In the presence of EPI-NCSC grafts, the rostral spread of the injury was significantly diminished on the left side only,
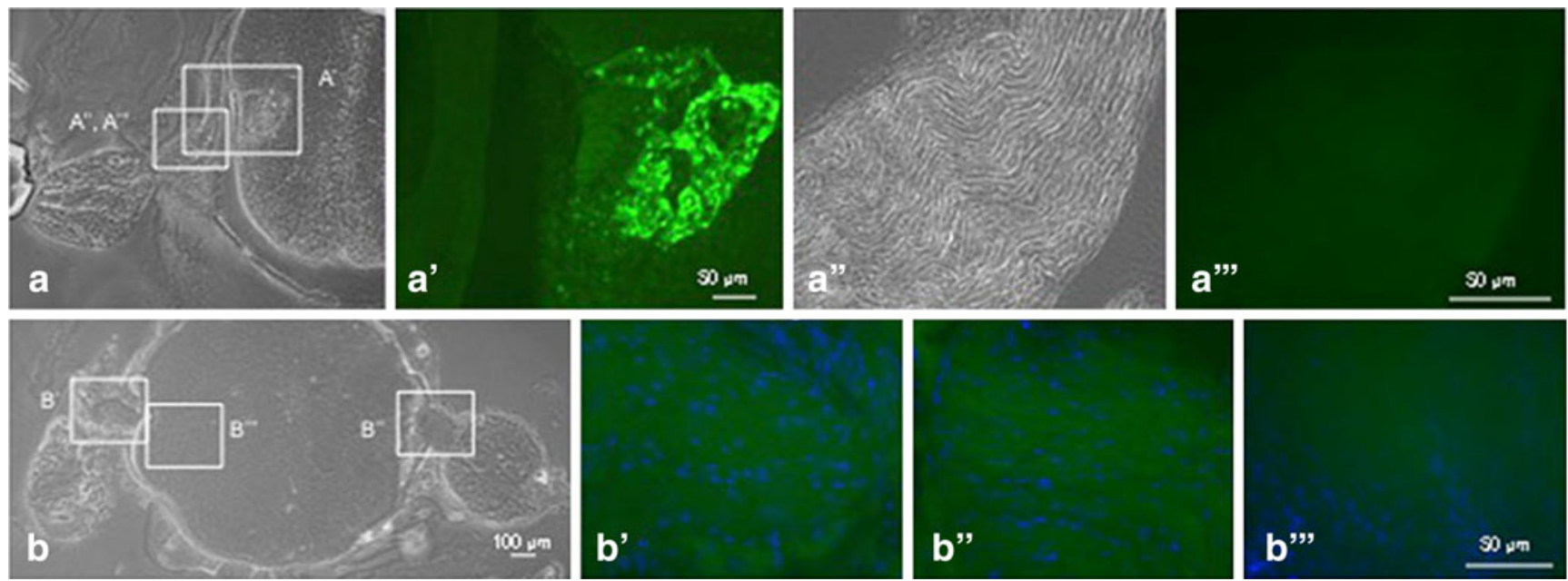

Fig. 8 Absence of elements of grafted cells in spinal roots and ganglia. Cross sections of spinal cords containing EPI-NCSC grafts were inspected at the level of the graft and at several segments above and below the graft to determine whether intraspinal EPI-NCSC . (a A"') show the level of the graft. a, phase contrast optics; A', higher magnification of boxed area in a; green fluorescent grafted cells are present in the spinal cord, but not in the dorsal root. A", A"; higher magnification of area boxed in A labeled A", A" showing a section of the dorsal and ventral roots in phase contrast optics (A") and green fluorescence (A"'). No EPI-NCSC derived cell or fibers are present. (b-B"') Section of same spinal cord as in a with EPI-NCSC graft, three segments above the site of grafting. b, phase contrast optics. B', green fluorescence image with DAPI stain of boxed area in $\mathbf{b}$, which shows the left dorsal root. No fluorescent cells or fibers are present. B", green fluorescence of contra lateral dorsal root. No EPI-NCSCderived cells or fibers are present. B"'; area of lesion three segments below in green fluorescence illumination. No EPI-NCSC are present at that level 
Table 2 Expression levels of growth factors, Angiogenic factors and proteases

Ratio gene/average three housekeeping genes (mean \pm S.E.M.)

$\begin{array}{lr}\text { Growth factors } & 86.3 \pm 4.0 \\ \text { Nerve growth factor (Ngf) } & 80.6 \pm 3.7 \\ \text { Brain derived neurotrophic factor (Bdnf) } & 79.2 \pm 3.6 \\ \text { Fibroblast growth factor-2 (Fgf2) } & 89.9 \pm 3.8 \\ \text { Fibroblast growth factor-7 (Fgf7) } & 70.9 \pm 3.4 \\ \text { Transforming growth factor-2 (Tgfb2) } & 75.9 \pm 3.5 \\ \text { Insulin-like growth factor-2 (Igf2) } & 82.9 \pm 2.9 \\ \text { Bone morphogenetic protein-1 (Bmp1) } & 66.0 \pm 3.1 \\ \text { Bone morphogenetic protein-2 (Bmp2) } & 93.9 \pm 2.4 \\ \text { Angiogenic factors } & 83.5 \pm 3.3 \\ \text { Vascular endothelial growth factor A (Vegfa) } & \\ \text { Vascular endothelial growth factor B (Vegfb) } & 97.9 \pm 3.4 \\ \text { Metalloproteases } & 92.4 \pm 4.9 \\ \text { Matrix metallopeptidase-2(Mmp2) } & 94.2 \pm 2.6 \\ \text { Matrix metallopeptidase-3 (Mmp3) } & 78.8 \pm 2.7 \\ \text { Matrix metallopeptidase-14 (Mmp14) } & \\ \text { A disintegrin-like and metallopeptidase (reprolysin type) } & \\ \quad \text { with thrombospondin type 1 motif (Adamts1) } & \end{array}$

Individual expression levels of housekeeping genes were as follows; Gapdh, $94.5 \pm 3.5$; $\beta$-actin, $105.1 \pm 2.6$; RPS9 (ribosomal protein S9), $108.7 \pm 2.8$

suggesting that the mechanism of action is cellular, such as the observed remyelination. While the current effect is small, it warrants further investigation in future studies.

Since the contusion was applied to the dorsal column, the SpSEPs recorded in the current study may have originated from action potentials in the lateral spinocerebellar or spinothalamic tracts. Of these tracts, the spinocerebellar tract is expected to have a larger contribution to the SpSEP since the stimulus primarily activated large diameter sensory fibers, and spinothalamic tracts are more generally associated with pain and temperature sensation (i.e. high-threshold afferents). In addition, mid-latency evoked potentials of the mouse have previously been associated with spinocerebellar pathways [28], which are located within the lateral tracts of the spinal cord. These tracts, which carry proprioceptive information from large diameter afferents, were more likely to be spared by the contusion injury in the current study. Thus, the retention of SpSEPs with EPI-NCSC transplants might have reflected a relative sparing of the spinocerebellar tracts. This would be consistent with a neuroprotective effect of the EPINCSC. Despite this functional improvement, allodynia was not observed 4 months post-injury/surgery.

An alternative explanation for the larger SpSEPs and lower Semmes-Weinstein threshold is an increased excitability of the lumbosacral spinal cord to afferent drive in EPI-NCSC transplanted mice. An increased caudal spinal cord excitability could produce a larger ascending action potential. After a spinal cord injury, it is common for the excitability of spinal reflexes to increase, as reflected by lower thresholds for $\mathrm{H}$ reflexes in spinal contused rats [29] and decreases in rate depression in spinal transected rats [30]. A generalized hyperexcitability of spinal cord neurons in chronic injury has also been documented in rats using fMRI [31]. Further, the clonus observed in the EPI-NCSC transplanted mice is analogous to clonus in humans [32], which occurs in nearly $70 \%$ of patients with spinal cord injury [33]. While reflex excitability was not directly measured in the current study, observations of clonus are consistent with greater spinal excitability in the EPI-NCSC transplanted mice. The observation of a larger SpSEP and lower sensory threshold might be the result an increased excitability of the lumbosacral spinal cord, which could result in an increased synaptic drive to spinocerebellar tracts, thereby increasing SpSEP amplitude.

EPI-NCSC express several metalloproteinases and other extracellular proteases. There is an intriguing parallel between the MRL/MpJ mouse and neural crest cells, and thus EPINCSC, which may be pertinent to the beneficial effects of EPINCSC grafts in this model of spinal cord injury. The MRL/ $\mathrm{MpJ}$ mouse has extraordinary regenerative capacity as has been shown for instance in an ear hole model [34]. This observation has been ascribed to the expression of high levels of matrix metalloproteinases and has been considered causative to tissue regeneration [34]. As invasive migratory cells that need to translocate through the extracellular matrix 
in the embryo to reach their target locations, it is an inherent property of neural crest cells to express matrix-degrading enzymes. We have shown in this study with a few examples that EPI-NCSC indeed express metalloproteases and other extracellular proteases. Timp-2 inhibits Mmp-2. The ratio of Mmp-2/Timp-2 in EPI-NCSC is 1.5 according to the LongSAGE gene expression profile [15]. This value equals or exceeds that in MRL/MpJ mice (1.09-1.6; [34]), indicating a high level of metalloproteinase activity in the microenvironment of EPI-NCSC. Elevated levels of metalloproteases alone, however, cannot be responsible for functional improvement, as has been shown in the MRL/MpJ mouse [35]. We nevertheless can conclude that metalloproteases released by EPI-NCSC are likely to participate actively in the remodeling of extracellular matrix during scar formation and thus potentially reduce the barrier effect of the scar. Proteases may also promote the release of relevant membrane-bound growth factors in host cells and/or grafted cells.

In summary, EPI-NCSC, unlike other types of stem cell, combine several desirable features that can be causative to improved sensory connectivity and touch perception in this mouse model of spinal cord injury. While there are some differences between murine and human EPI-NCSC (Clewes et al, unpublished), and mouse models are not ideal to assess human conditions, our present study nevertheless shows that EPI-NCSC are attractive candidates for the treatment of spinal cord injury, as they combine a number of aspects relevant to cell-based combination therapy.

\begin{abstract}
Acknowledgements The study was supported by the North East England Stem Cell Institute, Newcastle University (MSB); Biomedical Technology Alliance of Southeastern Wisconsin (MSB, BDS); Bryon Riesch Paralysis Foundation, Milwaukee (MSB); Fraternal Order of Eagles Midwest Chapter (MSB); National Spinal Cord Injury Association Milwaukee Chapter (MSB) and Falk Medical Research Trust (BDS). MSB thanks Martin E Schwab for stimulating discussions and valuable advice and Bernd Fritsch for advice on administering retrograde tracer.
\end{abstract}

Conflict of interest statement None of the authors have any conflicts of interest.

Open Access This article is distributed under the terms of the Creative Commons Attribution Noncommercial License which permits any noncommercial use, distribution, and reproduction in any medium, provided the original author(s) and source are credited.

\section{References}

1. Sieber-Blum, M., Grim, M., Hu, Y. F., \& Szeder, V. (2004). Pluripotent neural crest stem cells in the adult hair follicle. Dev Dyn., 231(2), 258-69.

2. Sieber-Blum, M., \& Grim, M. (2004). The adult hair follicle: cradle for pluripotent neural crest stem cells. Birth Defects Res C Embryo Today, 72(2), 162-72.
3. Mujtaba, T., Mayer-Proschel, M., \& Rao, M. S. (1998). A common neural progenitor for the CNS and PNS. Dev Biol., 200, 1-15.

4. Sieber-Blum, M., Schnell, L., Grim, M., Hu, Y. F., Schneider, R., \& Schwab, M. E. (2006). Characterization of epidermal neural crest stem cell (EPI-NCSC) grafts in the lesioned spinal cord. $\mathrm{Mol}$ Cell Neurosci., 32(1-2), 67-81. Epub 2006 Apr 19.

5. Ronsyn, M. W., Berneman, Z. N., Van Tendeloo, V. F. I., Jorens, P. G., \& Ponsaerts, P. (2008). Can cell therapy heal spinal cord injury? Spinal Cord, 46, 532-9.

6. Maier, I. C., Ichiyama, R. M., Courtine, G., Schnell, L., Lavrov, I., Edgerton, V. R., et al. (2009). Differential effects of anti-Nogo-A antibody treatment and treadmill training in rats with incomplete spinal cord injury. Brain., 132(Pt 6), 1426-40. Epub 2009 Apr 16.

7. Raisman, G. (2001). Olfactory ensheathing cells - another miracle cure for spinal cord injury? Nat Rev Neurosci., 2, 369-74.

8. Lindsay, S. L., Riddell, J. S., \& Barnett, S. C. (2010). Olfactory mucosa for transplant-mediated repair: a complex tissue for a complex injury? Glia, 58(2), 125-34.

9. Dasari, V. R., Spomar, D. G., Li, L., Gujrati, M., Rao, J. S., \& Dinh, D. H. (2008). Umbilical cord blood stem cell mediated downregulation of fas improves functional recovery of rats after spinal cord injury. Neurochem Res., 33(1), 134-49. Epub 2007 Aug 17.

10. Cho, J. S., Park, H. W., Park, S. K., Roh, S., Kang, S. K., Paik, K. S., et al. (2009). Transplantation of mesenchymal stem cells enhances axonal outgrowth and cell survival in an organotypic spinal cord slice culture. Neurosci Lett., 454(1), 43-8. Epub 2009 Feb 20.

11. Carter, L. M., Starkey, M. L., Akrimi, S. F., Davies, M., McMahon, S. B., \& Bradbury, E. J. (2008). The yellow fluorescent protein (YFP-H) mouse reveals neuroprotection as a novel mechanism underlying chondroitinase $\mathrm{ABC}$-mediated repair after spinal cord injury. J Neurosci., 28(52), 14107-20.

12. Hu X, Cai J, Yang J, Smith GM. (2009). Sensory axon targeting is increased by NGF gene therapy within the lesioned adult femoral nerve. Exp Neurol. 2009 Sep 4.

13. Alto, L. T., Havton, L. A., Conner, J. M., Hollis Ii, E. R., Blesch, A., \& Tuszynski, M. H. (2009). Chemotropic guidance facilitates axonal regeneration and synapse formation after spinal cord injury. Nat Neurosci., 12(9), 1106-13. Epub 2009 Aug 2.

14. Okabe, M., Ikawa, M., Kominami, K., Nakanishi, T., \& Nishimune, Y. (1997). 'Green mice' as a source of ubiquitous green cells. FEBS Lett., 407, 313-9.

15. Hu, Y. F., Zhang, Z. J., \& Sieber-Blum, M. (2006). An epidermal neural crest stem cell (EPI-NCSC) molecular signature. Stem Cells., 24(12), 2692-702 Epub 2006 Aug 24.

16. Sieber-Blum M, Hu YF. Mouse epidermal neural crest stem cell (EPI-NCSC) cultures. (2008b) J Vis Exp. 9(15). pii: 772. doi: $10.3791 / 772$.

17. Ellingson, B. M., Kurpad, S. N., \& Schmit, B. D. (2008). Characteristics of mid- to long-latency spinal somatosensory evoked potentials following spinal trauma in the rat. $J$ Neurotrauma., 25(11), 1323-34.

18. Latash, M. L. (1988). "Early" and "late" components of spinal evoked potentials in spinal cord trauma patients. Electromyogr Clin Neurophysiol., 28(4), 175-81.

19. Ameye, L., Hermann, R., Killian, C., Wilt, F., \& Dubois, P. (1999). Ultrastructural localization of proteins involved in sea urchin biomineralization. J Histochem Cytochem., 47(9), 1189-200.

20. Fritzsch, B. (1993). Fast axonal diffusion of 3000 molecular weight dextran amines. J Neurosci Methods., 50(1), 95-103.

21. Sieber-Blum, M., \& Hu, Y. F. (2008). Epidermal neural crest stem cells (EPI-NCSC) and pluripotency. Stem Cell Rev and Reports., 4, 256-60.

22. Valeriani, M., Le Pera, D., Restuccia, D., de Armas, L., Miliucci, R., Betti, V., et al. (2007). Parallel spinal pathways generate the middle-latency N1 and the late P2 components of the laser evoked potentials. Clin Neurophysiol., 118(5), 1097-104. 
23. Chung, J. M., Kenshalo, D. R., Jr., Gerhart, K. D., \& Willis, W. D. (1979). Excitation of primate spinothalamic neurons by cutaneous C-fiber volleys. J Neurophysiol., 42(5), 1354-69.

24. Kenshalo, D. R., Jr., Giesler, G. J., Jr., Leonard, R. B., \& Willis, W. D. (1980). Responses of neurons in primate ventral posterior lateral nucleus to noxious stimuli. J Neurophysiol., 43(6), 1594-614.

25. Howard, M. J., \& Gershon, M. D. (1993). Role of growth factors in catecholaminergic expression by neural crest cells: in vitro effects of transforming growth factor beta 1. Dev Dyn., 196, 1-10.

26. Zhang, J. M., Hoffmann, R. M., \& Sieber-Blum, M. (1997). Mitogenic and anti-proliferative signals for neural crest cells and the neurogenic action of TGF-b1. Dev Biol., 208, 375-86.

27. Hooshmand, M. J., Sontag, C. J., Uchida, N., Tamaki, S., Anderson, A. J., \& Cummings, B. J. (2009). Analysis of hostmediated repair mechanisms after human CNS-stem cell transplantation for spinal cord injury: correlation of engraftment with recovery. PLoS One, 4(6), e5871.

28. Chandran, A. P., Oda, K., Shibasaki, H., \& Pisharodi, M. (1994). Spinal somatosensory evoked potentials in mice and their developmental changes. Brain Dev., 16(1), 44-51.

29. Thompson, F. J., Reier, P. J., Lucas, C. C., \& Parmer, R. (1992). Altered patterns of reflex excitability subsequent to contusion injury of the rat spinal cord. $J$ Neuropsychiatry, 68(5), 1473-86.
30. Yates, C., Charlesworth, A., Allen, S. R., Reese, N. B., Skinner, R. D., \& Garcia-Rill, E. (2008). The onset of hyperreflexia in the rat following complete spinal cord transection. Spinal Cord, 46 (12), 798-803.

31. Endo, T., Spenger, C., Westman, E., Tominaga, T., \& Olson, L. (2008). Reorganization of sensory processing below the level of spinal cord injury as revealed by fMRI. Exptl Neurol., 209, 15560.

32. Benz, E. N., Hornby, T. G., Bode, R. K., Scheidt, R. A., \& Schmit, B. D. (2005). A physiologically based clinical measure for spastic reflexes in spinal cord injury. Arch Phys Med Rehabil., 86(1), 52-9.

33. Fleuren, J. F., Voerman, G. E., Snoek, G. J., Nene, A. V., Rietman, J. S., \& Hermens, H. J. (2009). Perception of lower limb spasticity in patients with spinal cord injury. Spinal Cord, 47(5), 396-400.

34. Gourevitch, D., Clark, L., Chen, P., Seitz, A., Samulewicz, S. J., \& Heber-Katz, E. (2003). Matrix metalloproteinase activity correlates with blastema formation in the regenerating MRL mouse ear hole model. Dev Dyn., 226, 377-87.

35. Hampton, D. W., Seitz, A., Chen, P., Heber-Katz, E., \& Fawcett, J. W. (2004). Altered CNS response to injury in the MRL/MpJ mouse. Neuroscience., 127(4), 821-32. 